\title{
GRAVITATIONAL BINARY-LENS EVENTS WITH PROMINENT EFFECTS OF LENS ORBITAL MOTION
}

\author{
H. PARK ${ }^{1,33}$, A. UdAlski ${ }^{2,34}$, C. HAN ${ }^{1,33,35}$, A. Gould ${ }^{3,33}$, J.-P. BEAulied ${ }^{4,36}$, Y. TsaPras ${ }^{5,6,37}$, M. K. SzYMAŃSKI ${ }^{2}$, M. KubiaK $^{2}$, \\ I. Soszyński ${ }^{2}$, G. Pietrzyński ${ }^{2,7}$, R. Poleski ${ }^{2,3}$, K. UlaczyK $^{2}$, P. Pietrukowicz $^{2}$, S. KozłowsKi $^{2}$, \\ J. SKOWRON ${ }^{2}$, Ł. WYRZYKOWSKI ${ }^{2,8}$ \\ (The OGLE COLlaboration) \\ J.-Y. Choi ${ }^{1}$, D. L. Depoy ${ }^{9}$, Subo Dong ${ }^{10}$, B. S. Gaudi ${ }^{3}$, K.-H. Hwang ${ }^{1}$, Y. K. Jung ${ }^{1}$, A. KavkA ${ }^{3}$, C.-U. LeE ${ }^{11}$, \\ L. A. G. Monard ${ }^{12}$, B.-G. Park ${ }^{11}$, R. W. Pogge ${ }^{3}$, I. Porritt ${ }^{13}$, I.-G. Shin ${ }^{1}$, J. C. YeE ${ }^{3}$ \\ (The $\mu$ FUN Collaboration) \\ M. D. Albrow ${ }^{14}$, D. P. Bennett ${ }^{15}$, J. A. R. Caldwell ${ }^{16}$, A. Cassan ${ }^{4}$, C. Coutures ${ }^{4}$, D. Dominis ${ }^{17}$, J. Donatowicz ${ }^{18}$, \\ P. Fouqué ${ }^{19}$, J. Greenhill ${ }^{20}$, M. Huber ${ }^{21}$, U. G. Jørgensen ${ }^{22}$, S. KAne ${ }^{23}$, D. Kubas ${ }^{4}$, J.-B. Marquette ${ }^{4}$, J. Menzies ${ }^{24}$, \\ C. Pitrou ${ }^{4}$, K. R. Pollard ${ }^{14}$, K. C. Sahu ${ }^{25}$, J. Wambsganss ${ }^{26}$, A. Williams ${ }^{27}$, M. Zub ${ }^{26}$ \\ (The Planet COLlaboration) \\ A. Allan $^{28}$, D. M. Bramich ${ }^{29}$, P. Browne ${ }^{30}$, M. DominiK $^{30}$, K. Horne ${ }^{30}$, M. Hundertmark ${ }^{30}$, N. Kains $^{29}$, \\ C. Snodgrass ${ }^{31}$, I. A. Steele ${ }^{32}$, and R. A. Street $^{5}$ \\ (The RoboNet Collaboration) \\ ${ }^{1}$ Department of Physics, Institute for Astrophysics, Chungbuk National University, Cheongju 371-763, Korea \\ ${ }^{2}$ Warsaw University Observatory, Al. Ujazdowskie 4, 00-478 Warszawa, Poland \\ ${ }^{3}$ Department of Astronomy, The Ohio State University, 140 West 18th Avenue, Columbus, OH 43210, USA \\ ${ }^{4}$ Institut d'Astrophysique de Paris, UMR 7095 CNRS-Université Pierre \& Marie Curie, 98bis Bd Arago, F-75014 Paris, France \\ ${ }^{5}$ Las Cumbres Observatory Global Telescope Network, 6740 Cortona Drive, Suite 102, Goleta, CA 93117, USA \\ ${ }^{6}$ School of Mathematical Sciences, Queen Mary, University of London, Mile End Road, London E1 4NS, UK \\ ${ }^{7}$ Universidad de Concepción, Departamento de Astronomia, Casilla 160-C, Concepción, Chile \\ ${ }^{8}$ Institute of Astronomy, University of Cambridge, Madingley Road, Cambridge CB3 0HA, UK \\ ${ }^{9}$ Department of Physics and Astronomy, Texas A\&M University, College Station, TX 77843, USA \\ ${ }^{10}$ Institute for Advanced Study, Einstein Drive, Princeton, NJ 08540, USA \\ ${ }^{11}$ Korea Astronomy and Space Science Institute, 776 Daedukdae-ro, Yuseong-gu, Daejeon 305-348, Korea \\ ${ }^{12}$ Klein Karoo Observatory, Calitzdorp, and Bronberg Observatory, Pretoria, South Africa \\ ${ }^{13}$ Turitea Observatory, Palmerston North, New Zealand \\ ${ }^{14}$ Department of Physics and Astronomy, University of Canterbury, Private Bag 4800, Christchurch 8020, New Zealand \\ ${ }^{15}$ Department of Physics, University of Notre Dame, 225 Nieuwland Science Hall, Notre Dame, IN 46556-5670, USA \\ ${ }^{16}$ McDonald Observatory, University of Texas, Fort Davis, TX 79734, USA \\ ${ }^{17}$ Astrophysikalisches Institut Potsdam, An der Sternwarte 16, 14482 Potsdam, Germany \\ ${ }^{18}$ Department of Computing, Technical University of Vienna, Wiedner Hauptstrasse 10, A-1040 Vienna, Austria \\ ${ }^{19}$ Observatoire Midi-Pyrénées, Laboratoire d'Astrophysique, UMR 5572, Université Paul Sabatier-Toulouse 3, 14 avenue Edouard Belin, 31400 Toulouse, France \\ ${ }^{20}$ School of Mathematics and Physics, University of Tasmania, Private Bag 37, Hobart, TAS 7001, Australia \\ ${ }^{21}$ Institute for Astronomy, University of Hawaii, 2680 Woodlawn Drive Honolulu, HI 96822-1839, USA \\ ${ }^{22}$ Niels Bohr Institute, Astronomical Observatory, Juliane Maries vej 30, 2100 Copenhagen, Denmark \\ ${ }^{23}$ NASA Exoplanet Science Institute, Caltech, MS 100-22, 770 South Wilson Avenue, Pasadena, CA 91125, USA \\ ${ }^{24}$ South African Astronomical Observatory, P.O. Box 9 Observatory 7935, South Africa \\ ${ }^{25}$ Space Telescope Science Institute, 3700 San Martin Drive, Baltimore, MD 21218, USA \\ ${ }^{26}$ Astronomisches Rechen-Institut (ARI), Zentrum für Astronomie der Universität Heidelberg (ZAH), Mönchhofstr. 12-14, D-69120, Heidelberg, Germany \\ ${ }^{27}$ Perth Observatory, Walnut Road, Bickley, Perth 6076, Australia \\ ${ }^{28}$ School of Physics, University of Exeter Stocker Road, Exeter, Devon, EX4 4QL, UK \\ ${ }^{29}$ European Southern Observatory, Karl-Schwarzschild-Straße 2, D-85748 Garching bei München, Germany \\ ${ }^{30}$ SUPA, University of St. Andrews, School of Physics and Astronomy, North Haugh, St. Andrews, KY16 9SS, UK \\ ${ }^{31}$ Max Planck Institute for Solar System Research, Max-Planck-Str. 2, D-37191 Katlenburg-Lindau, Germany \\ ${ }^{32}$ Astrophysics Research Institute, Liverpool John Moores University, Twelve Quays House, Egerton Wharf, Birkenhead, Wirral., CH41 1LD, UK \\ Received 2013 June 17; accepted 2013 October 4; published 2013 November 12
}

\begin{abstract}
Gravitational microlensing events produced by lenses composed of binary masses are important because they provide a major channel for determining physical parameters of lenses. In this work, we analyze the light curves of two binary-lens events, OGLE-2006-BLG-277 and OGLE-2012-BLG-0031, for which the light curves exhibit strong deviations from standard models. From modeling considering various second-order effects, we find that the deviations are mostly explained by the effect of the lens orbital motion. We also find that lens parallax effects can mimic orbital effects to some extent. This implies that modeling light curves of binary-lens events not considering orbital effects can result in lens parallaxes that are substantially different from actual values and thus wrong determinations of physical lens parameters. This demonstrates the importance of routine consideration of orbital effects in interpreting light curves of binary-lens events. It is found that the lens of OGLE-2006-BLG-277 is a binary composed of a low-mass star and a brown dwarf companion.
\end{abstract}

Key words: binaries: general - gravitational lensing: micro - planets and satellites: general

Online-only material: color figures 


\section{INTRODUCTION}

Progress in gravitational microlensing experiments over the last two decades has enabled a great increase in the number of event detections from tens of events per year at the early stage to several thousands per year in current experiments. Among discovered lensing events, an important portion are produced by lenses composed of two masses (Mao \& Paczyński 1991).

One reason why binary-lens events are important is that these events provide a major channel for determining the physical parameters of lenses. To determine lens parameters from observed lensing light curves, one must simultaneously measure the lens parallax $\pi_{\mathrm{E}}$ and the angular Einstein radius $\theta_{\mathrm{E}}$. The lens parallax is measured from long-term deviations in lensing light curves caused by the positional change of an observer induced by the orbital motion of the Earth around the Sun: the parallax effect (Gould 1992). The Einstein radius, however, is measured from deviations in lensing light curves affected by the finite size of a source star: the finite-source effect (Gould 1994; Witt \& Mao 1994). With the measured values of $\pi_{\mathrm{E}}$ and $\theta_{\mathrm{E}}$, the mass and distance to the lens are determined, respectively, by

$$
M_{\mathrm{tot}}=\frac{\theta_{\mathrm{E}}}{\kappa \pi_{\mathrm{E}}} ; \quad D_{\mathrm{L}}=\frac{\mathrm{AU}}{\pi_{\mathrm{E}} \theta_{\mathrm{E}}+\pi_{\mathrm{S}}},
$$

where $\kappa=4 G /\left(c^{2} \mathrm{AU}\right), \mathrm{AU}$ is an astronomical unit, $\pi_{\mathrm{S}}=$ $\mathrm{AU} / D_{\mathrm{S}}$, and $D_{\mathrm{S}}$ is the distance to the lensed star (Gould 1992; Gould et al. 2006). For single-lens events, the chance to measure $\theta_{\mathrm{E}}$ is very low because finite-source effects occur only for very rare events with extremely high magnifications in which the lens passes over the surface of the source star, e.g., Choi et al. (2012). By contrast, the chance to measure $\theta_{\mathrm{E}}$ is high for binary-lens events because most of these events involve source stars' caustic crossings or approaches during which finite-source effects are important. As a result, the majority of gravitational lenses with measured physical parameters are binaries.

It is known that changes of lens positions caused by the orbital motion of a binary lens can induce long-term deviations in lensing light curves, similar to deviations induced by parallax effects. Since this was first detected for the event MACHO97-BLG-41 (Bennett et al. 1999; Albrow et al. 2000; Jung et al. 2013), orbital effects have been considered for more binary-lens events (e.g., An et al. 2002; Jaroszyński et al. 2005; Skowron et al. 2011; Shin et al. 2011, 2013). However, analyses have been carried out only for a limited number of events. An important obstacle to orbital analyses is the heavy computation required to consider the time variation of the caustic morphology caused by the orbit-induced changes of the binary separation and orientation. As a result, routine orbital analyses for general binary-lens events became possible very recently after being able to utilize efficient modeling software and powerful computing resources.

Considering orbital effects is important for accurate determinations of physical lens parameters. Since orbital and parallax effects induce similar long-term deviations, it might be that orbital effects can be mimicked by parallax effects. Then, if only parallax effects are considered for events affected by orbital

\footnotetext{
33 The $\mu$ FUN Collaboration.

34 The OGLE Collaboration.

${ }^{35}$ Corresponding author.

36 The PLANET Collaboration.

37 The RoboNet Collaboration.
}

Table 1

Telescopes

\begin{tabular}{ll}
\hline \hline Event & \multicolumn{1}{c}{ Telescopes } \\
\hline OGLE-2006-BLG-277 & OGLE, 1.3 m Warsaw, LCO, Chile \\
& $\mu$ FUN, $1.3 \mathrm{~m}$ SMARTS, CTIO, Chile \\
& PLANET, $1.5 \mathrm{~m}$ Boyden, South Africa \\
& PLANET, $1.0 \mathrm{~m}$ Canopus, Australia \\
& PLANET, $0.6 \mathrm{~m}$ Perth, Australia \\
& PLANET, $1.54 \mathrm{~m}$ Danish, Chile \\
& RoboNet, $2.0 \mathrm{~m}$ LT, La Palma, Spain \\
\hline OGLE-2012-BLG-0031 & OGLE, $1.3 \mathrm{~m}$ Warsaw, LCO, Chile \\
& $\mu$ FUN, $1.3 \mathrm{~m}$ SMARTS, CTIO, Chile \\
& $\mu$ FUN, $0.36 \mathrm{~m}$ Turitea, New Zealand \\
& $\mu$ FUN, 0.36 m KKO, South Africa \\
& RoboNet, 2.0 m FTS, Australia \\
& RoboNet, $2.0 \mathrm{~m}$ LT, La Palma, Spain \\
\hline
\end{tabular}

Notes. LCO: Las Campanas Observatory, CTIO: Cerro Tololo Inter-American Observatory, KKO: Klein Karoo Observatory, LT: Liverpool Telescope, FTS: Faulkes Telescope South.

effects, the determined physical parameters would be different from their true values. In this work, we demonstrate the importance of considering orbital effects by presenting analyses of two binary-lens events.

\section{OBSERVATION}

The events analyzed in this work are OGLE-2006-BLG-277 and OGLE-2012-BLG-0031. Both events occurred on stars toward the Galactic bulge field with equatorial coordinates $(\alpha, \delta)_{\mathrm{J} 2000}=\left(18^{\mathrm{h}} 01^{\mathrm{m}} 14^{\mathrm{s}} .84,-27^{\circ} 48^{\prime} 36^{\prime \prime} \cdot 2\right)$, corresponding to the Galactic coordinates $(l, b)=(2.71,-2.39)$, for OGLE2006-BLG-277 and $(\alpha, \delta)_{\mathrm{J} 2000}=\left(17^{\mathrm{h}} 50^{\mathrm{m}} 50^{\mathrm{s}} 53,-29^{\circ} 10^{\prime} 48^{\prime \prime} .8\right)$, corresponding to $(l, b)=(0.38,-1.10)$, for OGLE-2012-BLG0031. The events were discovered from survey observations conducted by the Optical Gravitational Lensing Experiment (OGLE; Udalski 2003). In addition to the survey observation, the events were additionally observed by follow-up groups including the Probing Lensing Anomalies NETwork (PLANET; Albrow et al. 1998), the Microlensing Follow-Up Network ( $\mu$ FUN; Gould et al. 2006), and the RoboNet (Tsapras et al. 2009) groups. In Table 1, we list survey and follow-up groups who participated in observations of the individual events along with the telescopes they employed as well as their locations. We note that the event OGLE-2006-BLG-277 was previously analyzed by Jaroszyński et al. (2010), but the analysis was based on only OGLE data. We therefore reanalyze the event based on all combined data considering higher-order effects.

Data were reduced using photometry codes developed by the individual groups, mostly based on difference image analysis (Alard \& Lupton 1998; Woźniak et al. 2001; Bramich 2008; Albrow et al. 2009). In order to use data sets acquired from different observatories, we readjust the error bars. To do this, we first add a quadratic error term so that the cumulative distribution of $\chi^{2}$ ordered by magnifications is approximately linear in data counts, and then rescale errors so that $\chi^{2}$ per degree of freedom $\left(\chi^{2} /\right.$ dof $)$ becomes unity, i.e., $\sigma_{i}=k_{i}\left(\sigma_{0}^{2}+e_{i}^{2}\right)^{1 / 2}$, where $\sigma_{0}$ is the original error bar, $i$ is the index referring to each data set, $e_{i}$ is the quadratic error term, and $k_{i}$ is the rescaling function.

In Figures 1 and 2, we present the light curves of the individual events. Both light curves exhibit sharp spikes that are characteristic features of caustic-crossing binary-lens events. The spikes occur in pairs because the caustic forms a closed 


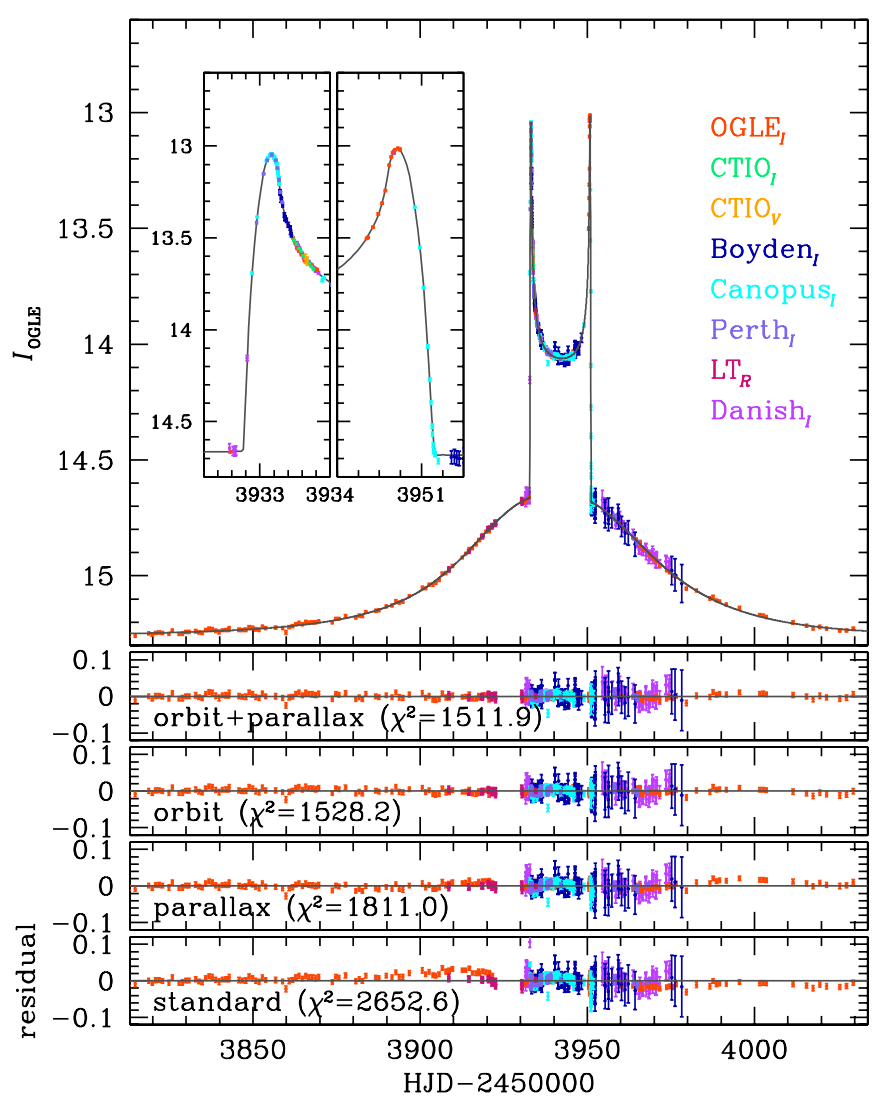

Figure 1. Light curve of OGLE-2006-BLG-277. In the legends indicating observatories, the subscript of each observatory denotes the passband. The subscript " $N$ " denotes that no filter is used. The insets in the upper panel show the enlargement of the caustic-crossing parts of the light curve. The lower four panels show the residuals of data from four different models.

(A color version of this figure is available in the online journal.)

curve. Usually, the inner region between two spikes has a "U"shape trough as in OGLE-2006-BLG-277. For OGLE-2012BLG-0031, the inner region exhibits a complex pattern. Such a pattern can be produced when the source trajectory runs approximately tangent to the fold of a caustic.

\section{MODELING}

\subsection{Standard Model}

Knowing that the events were produced by binary lenses, we conduct modeling of the observed light curves. Basic description of a binary-lens event requires seven lensing parameters. Among them, the first three parameters describe the lens-source approach. These parameters include the time of the closest approach of the source to a reference position ${ }^{38}$ of the binary lens, $t_{0}$, the lens-source separation at $t_{0}$ in units of the Einstein radius, $u_{0}$, and the time required for the source to cross the Einstein radius, $t_{\mathrm{E}}$ (Einstein time scale). The Einstein ring represents the source image for an exact lens-source alignment

\footnotetext{
38 For a binary lens with a projected separation less than the Einstein radius, $s<1$ (close binary), we set the reference position of the lens as the center of mass of the binary lens. For a binary with a separation greater than the Einstein radius, $s>1$ (wide binary), however, we set the reference as the photocenter that is located at a position with an offset $q /[s(1+q)]$ from the middle position between the two lens components. The photocenter represents a position in the source plane at which the contours of lensing magnification are centered. For a wide binary lens, two sets of widely separated caustics exist and the photocenter corresponds to the center of each caustic.
}

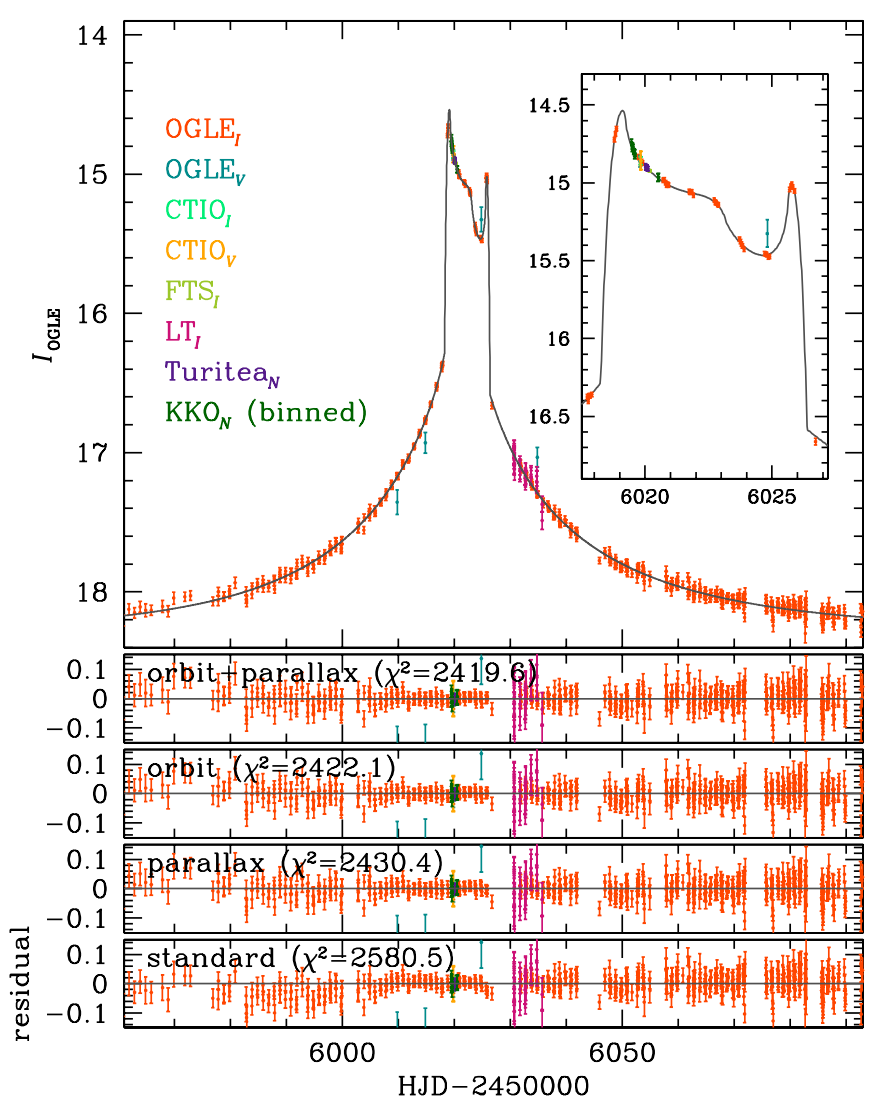

Figure 2. Light curve of OGLE-2012-BLG-0031. Notations are the same as those in Figure 1.

(A color version of this figure is available in the online journal.)

and its radius $\theta_{\mathrm{E}}$ is usually used as the length scale of lensing phenomena. The Einstein radius is related to the physical lens parameters by $\theta_{\mathrm{E}}=\left(\kappa M \pi_{\mathrm{rel}}\right)^{1 / 2}$, where $M$ is the mass of the lens and $\pi_{\text {rel }}=\mathrm{AU}\left(D_{\mathrm{L}}^{-1}-D_{\mathrm{S}}^{-1}\right)$ is the relative lens-source parallax. Another three lensing parameters describe the binary lens. These parameters include the projected separation, $s$ (in units of $\left.\theta_{\mathrm{E}}\right)$, the mass ratio between the binary lens components, $q$, and the angle between the source trajectory and the binary axis, $\alpha$ (source trajectory angle). The last parameter is the normalized source radius $\rho_{*}=\theta_{*} / \theta_{\mathrm{E}}$, where $\theta_{*}$ is the angular source radius. This parameter is needed to describe the parts of light curves affected by finite-source effects, which are important when a source star crosses over or approaches close to caustics formed by a binary lens.

In modeling the light curves based on the standard lensing parameters (standard model), searches for best-fit solutions have been done in two steps. In the first step, we identify local solutions by inspecting $\chi^{2}$ distributions in the parameter space. For this, we use both a grid search and a downhill approach. We choose $(s, q, \alpha)$ as grid parameters because lensing magnifications can vary dramatically with small changes in these parameters. By contrast, lensing magnifications vary smoothly with changes of the other parameters, and thus we search for the solutions of these parameters by minimizing $\chi^{2}$ using a downhill approach. We use the Markov Chain Monte Carlo (MCMC) method for the $\chi^{2}$ minimization. A thorough search in the grid parameter space is important to identify local minima for which different combinations of parameters result in a similar light curve. The range of grid parameters are set at $-1 \leqslant \log s \leqslant 1,-5 \leqslant \log q \leqslant 2$, and $0<\alpha<2 \pi$, which 

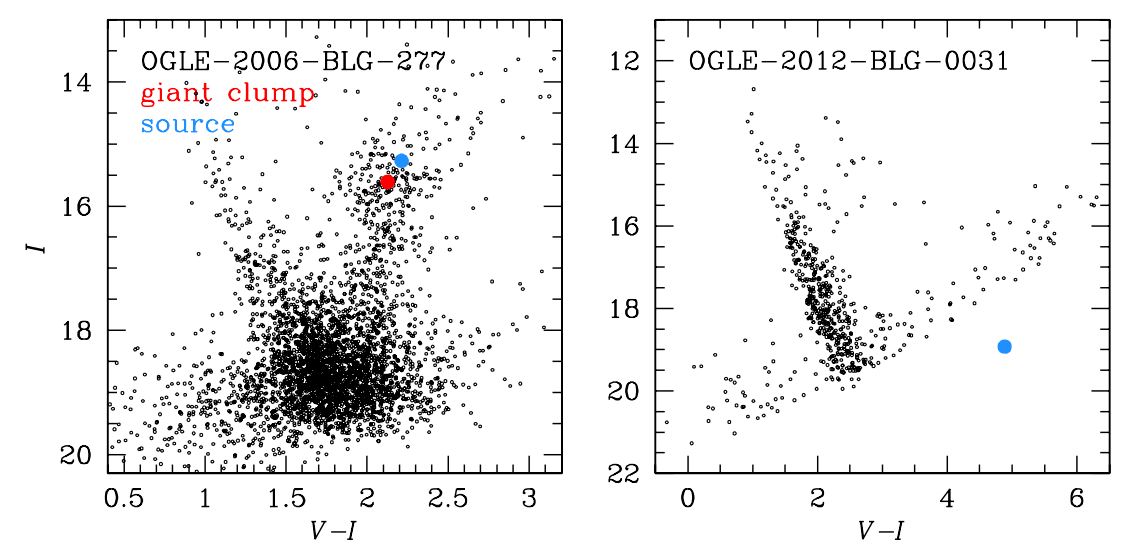

Figure 3. OGLE color-magnitude diagrams of the fields where OGLE-2006-BLG-277 (left panel) and OGLE-2012-BLG-0031 (right panel) occurred. The red and blue dots represent the centroid of the red giant clump and location of the lensed star, respectively.

(A color version of this figure is available in the online journal.)

are wide enough to encompass all possible local minima. In the second step, we refine the lensing parameters for the individual local solutions by allowing all parameters to vary. Then, the best-fit solution is obtained by comparing $\chi^{2}$ values of the individual local solutions. We estimate the uncertainties of the lensing parameters based on the distributions of the parameters obtained from the MCMC chain of solutions.

For magnification computations affected by finite-source effects, we use the "map-making method" developed by Dong et al. (2006). In this method, a map of rays for a given binary lens with a separation $s$ and a mass ratio $q$ is constructed by using the inverse ray-shooting technique (Schneider \& Weiss 1986; Kayser et al. 1986; Wambsganss 1997). In this technique, rays are uniformly shot from the image plane, bent according to the lens equation, and land on the source plane. The lens equation for a binary lens is represented by

$$
\zeta=z-\frac{m_{1}}{\bar{z}-\bar{z}_{\mathrm{L}, 1}}-\frac{m_{2}}{\bar{z}-\bar{z}_{\mathrm{L}, 2}}
$$

where $m_{1}=1 /(1+q)$ and $m_{2}=q m_{1}$ are the mass fractions of the individual binary lens components, $\zeta=\xi+i \eta, z=x+i y$, and $z_{\mathrm{L}, i}=x_{\mathrm{L}, i}+i y_{\mathrm{L}, i}$ denote the positions of the source, image, and lens expressed in complex notions, respectively, and $\bar{z}$ denotes the complex conjugate of $z$. With the constructed map, the finite-source magnification for a given position of a source with a normalized radius $\rho_{*}$ is computed as the ratio of the number density of rays within the source to that on the image plane. This method saves computation time by enabling one to produce many light curves resulting from various source trajectories based on a single map. In addition, the method enables one to speed up computation by allotting computation to multiple CPUs. We further accelerate computation by using semi-analytic hexadecapole approximation (Pejcha \& Heyrovský 2009; Gould 2008) for finite magnification computations.

In our finite-source computations, we consider the limbdarkening effect of the source star by modeling the surface brightness profile as

$$
S_{\lambda} \propto 1-\Gamma_{\lambda}\left(1-\frac{3}{2} \cos \phi\right),
$$

where $\Gamma_{\lambda}$ is the linear limb-darkening coefficient, $\lambda$ is the passband, and $\phi$ denotes the angle between the line of sight toward the source star and the normal to the source surface. The limbdarkening coefficients are adopted from Claret (2000) considering the source type that is determined based on the source locations in the color-magnitude diagrams (see Figure 3). We find that the source star of OGLE-2006-BLG-277 is a K-type giant star. For OGLE-2012-BLG-0031, the lensed star is located in a very reddened region, causing difficulties in precisely characterizing the star based on its color and brightness. Nevertheless, it is found that the source is a giant. The adopted coefficients are $\Gamma_{V}=0.74, \Gamma_{R}=0.64$, and $\Gamma_{I}=0.53$ for both events. For data sets obtained without any filter, we choose a mean value of the $R$ - and $I$-band coefficients, i.e., $\Gamma_{N}=\left(\Gamma_{R}+\Gamma_{I}\right) / 2$, where the subscript " $N$ " denotes that no filter is used.

In Figure 4, we present $\Delta \chi^{2}$ maps in the $(s, q)$ parameter space. For each event, the three panels show the maps in different ranges of parameters and contour levels where the upper panels show more refined maps. For OGLE-2006-BLG-277, we find a unique solution with no other local minimum. For OGLE2012-BLG-0031, however, several local minima exist. The pair of local minima with a similar $s$ but with opposite sign of $\log q$ simply implies mirror-image symmetry where $q<1$ represents the case where the heavier lens component is located on the left while it is located on the right for the $q>1$ case. A pair of local minima with a similar $q$ but with opposite signs of $\log s$ arises due to the well-known close/wide degeneracy (Griest \& Safidazeh 1998; Dominik 1999; An 2005). From detailed inspection of the local solutions including second-order effects (see Section 3.2), we find that the close binary solution $(s<1)$ provides a better fit than the wide solution $(s>1)$ with $\Delta \chi^{2} \sim 40(>6 \sigma)$.

In Table 2, we list the best-fit solutions of the lensing parameters obtained from standard modeling for the individual events. In Figures 1 and 2, we also present the residuals from the fits. It is found that even though the fits basically describe the main features of the observed light curves, important residuals exist that last throughout both events.

\subsection{Higher-order Effects}

Long-term residuals from the standard models suggest that one needs to consider higher-order effects in order to better describe the lensing light curves. Since it is known that such long-term residuals are caused by the parallax and/or lens orbital effects, we conduct additional modeling considering both higher-order effects. 
Table 2

Model Parameters

\begin{tabular}{|c|c|c|c|c|c|c|c|c|c|c|c|c|}
\hline Model & $\chi^{2} /$ dof & $\begin{array}{c}t_{0} \\
\left(\mathrm{HJD}^{\prime}\right)\end{array}$ & $u_{0}$ & $\begin{array}{c}t_{\mathrm{E}} \\
\text { (days) }\end{array}$ & $s$ & $q$ & $\alpha$ & $\begin{array}{c}\rho_{*} \\
\left(10^{-3}\right)\end{array}$ & $\pi_{\mathrm{E}, N}$ & $\pi_{\mathrm{E}, E}$ & $\begin{array}{l}d s / d t \\
\left(\mathrm{yr}^{-1}\right)\end{array}$ & $\begin{array}{l}d \alpha / d t \\
\left(\mathrm{yr}^{-1}\right)\end{array}$ \\
\hline \multicolumn{13}{|c|}{ OGLE-2006-BLG-277 } \\
\hline Standard & $2652.6 / 1499$ & $3941.620 \pm 0.020$ & $0.157 \pm 0.002$ & $39.13 \pm 0.08$ & $1.374 \pm 0.001$ & $2.600 \pm 0.037$ & $1.477 \pm 0.003$ & $5.83 \pm 0.02$ & & & & $\ldots$ \\
\hline Parallax only & $1811.0 / 1497$ & $3941.723 \pm 0.025$ & $0.169 \pm 0.003$ & $39.30 \pm 0.08$ & $1.371 \pm 0.001$ & $2.512 \pm 0.035$ & $1.485 \pm 0.003$ & $5.90 \pm 0.02$ & $0.45 \pm 0.07$ & $0.54 \pm 0.01$ & & $\ldots$ \\
\hline Orbit only & $1528.2 / 1497$ & $3943.066 \pm 0.031$ & $0.170 \pm 0.005$ & $38.78 \pm 0.07$ & $1.347 \pm 0.001$ & $2.033 \pm 0.030$ & $-1.485 \pm 0.005$ & $5.98 \pm 0.02$ & & & $0.73 \pm 0.02$ & $-0.33 \pm 0.11$ \\
\hline Orbit + parallax & $1511.9 / 1495$ & $3943.071 \pm 0.031$ & $-0.168 \pm 0.005$ & $37.90 \pm 0.13$ & $1.348 \pm 0.001$ & $1.981 \pm 0.030$ & $1.457 \pm 0.006$ & $6.03 \pm 0.02$ & $1.13 \pm 0.16$ & $-0.05 \pm 0.04$ & $0.69 \pm 0.03$ & $1.21 \pm 0.22$ \\
\hline \multicolumn{13}{|c|}{ OGLE-2012-BLG-0031 } \\
\hline Standard & $2580.5 / 2411$ & $6022.532 \pm 0.042$ & $0.046 \pm 0.001$ & $59.17 \pm 0.59$ & $0.477 \pm 0.003$ & $0.294 \pm 0.010$ & $0.800 \pm 0.009$ & $5.48 \pm 0.11$ & & & & $\ldots$ \\
\hline Parallax only & $2430.4 / 2409$ & $6022.233 \pm 0.043$ & $-0.047 \pm 0.001$ & $56.47 \pm 0.66$ & $0.510 \pm 0.003$ & $0.223 \pm 0.008$ & $-0.739 \pm 0.009$ & $5.63 \pm 0.11$ & $-0.29 \pm 0.08$ & $0.10 \pm 0.02$ & & \\
\hline Orbit only & $2422.1 / 2409$ & $6022.364 \pm 0.042$ & $0.051 \pm 0.001$ & $54.88 \pm 0.68$ & $0.511 \pm 0.003$ & $0.264 \pm 0.011$ & $0.774 \pm 0.009$ & $6.80 \pm 0.19$ & & $\ldots$ & $0.43 \pm 0.08$ & $3.63 \pm 0.20$ \\
\hline Orbit + parallax & $2419.6 / 2407$ & $6022.350 \pm 0.042$ & $-0.051 \pm 0.001$ & $54.58 \pm 0.77$ & $0.511 \pm 0.003$ & $0.268 \pm 0.010$ & $-0.773 \pm 0.009$ & $6.81 \pm 0.21$ & $-0.09 \pm 0.13$ & $0.03 \pm 0.02$ & $0.47 \pm 0.07$ & $-2.98 \pm 0.39$ \\
\hline
\end{tabular}

Note. HJD' $=$ HJD -2450000 . 

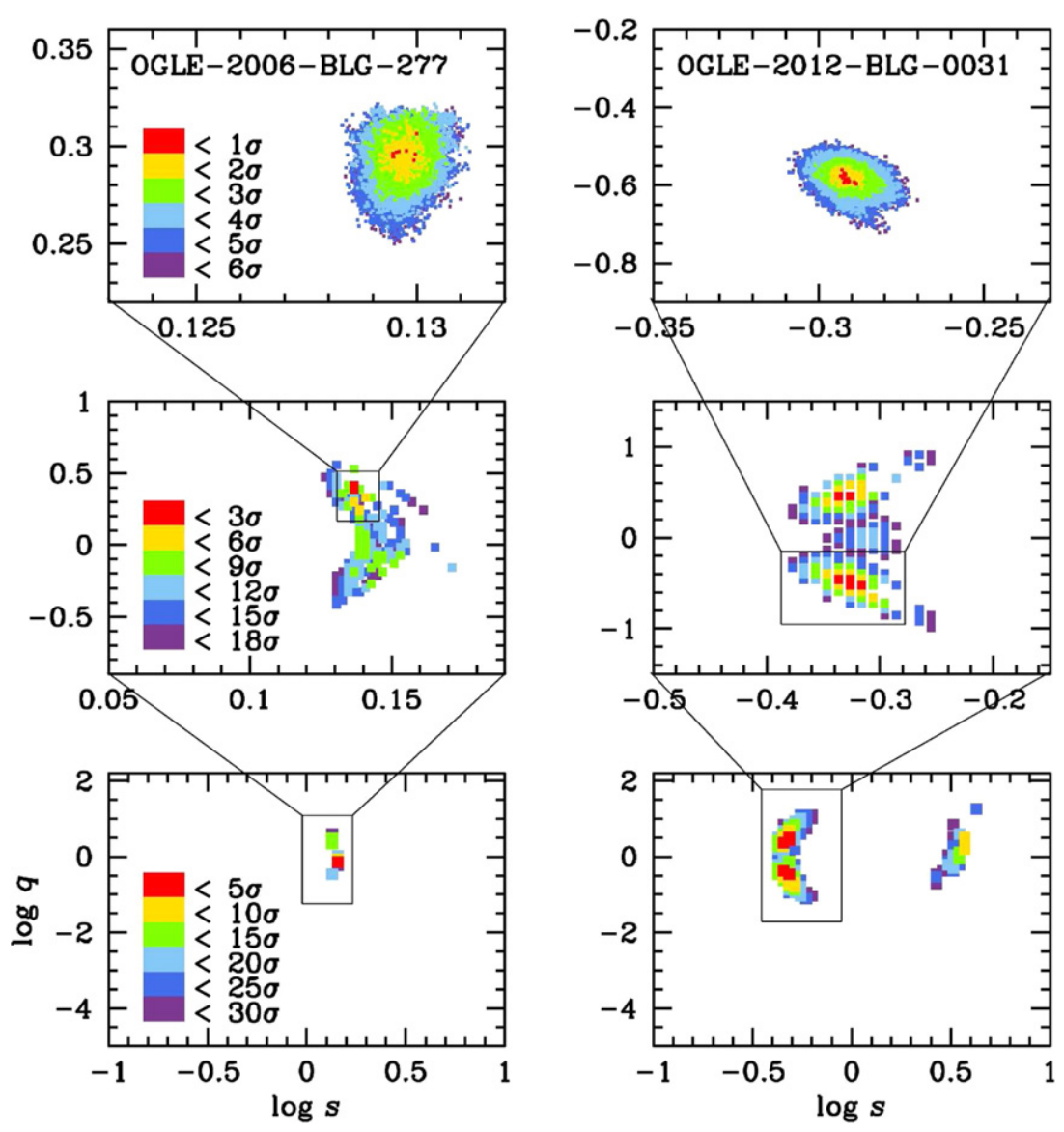

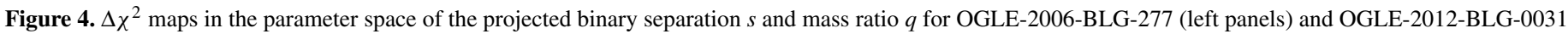

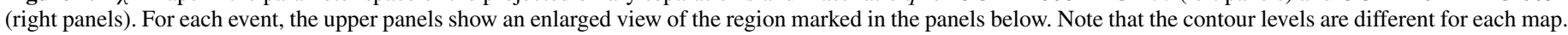
(A color version of this figure is available in the online journal.)

To describe parallax effects, it is necessary to include two parameters, $\pi_{\mathrm{E}, N}$ and $\pi_{\mathrm{E}, E}$, which represent the two components of the lens parallax vector $\pi_{\mathrm{E}}$ projected onto the sky along the north and east equatorial coordinates, respectively. The magnitude of the parallax vector, $\pi_{\mathrm{E}}=\left(\pi_{\mathrm{E}, N}^{2}+\pi_{\mathrm{E}, E}^{2}\right)^{1 / 2}$, corresponds to the relative lens-source parallax scaled to the Einstein radius of the lens, i.e., $\pi_{\mathrm{E}}=\pi_{\text {rel }} / \theta_{\mathrm{E}}$ (Gould 2004). The direction of the parallax vector corresponds to the relative lens-source motion in the frame of the Earth at a reference time of the event. In our modeling, we use $t_{0}$ as the reference time. Parallax effects cause the source motion relative to the lens to deviate from rectilinear.

To first-order approximation, the lens orbital motion is described by two parameters, $d s / d t$ and $d \alpha / d t$, which represent the change rates of the normalized binary separation and the source trajectory angle, respectively (Albrow et al. 2000; An et al. 2002). In addition to causing the relative lens-source motion to deviate from rectilinear, the orbital effect causes further deviation in lensing light curves by deforming the caustic over the course of the event due to the change of the binary separation.

In Table 2, we list the results of modeling considering the higher-order effects. For each event, we conduct three sets of additional modeling in which the parallax effect and orbital effect are considered separately ("parallax only" and "orbital only") and both effects are simultaneously considered ("orbit + parallax"). In the lower panels of Figures 1 and 2, we present the residuals of the individual models. In Figure 5, we present the geometry of the lens systems of the best-fit solutions, where the source trajectory with respect to the lens components and the resulting caustics are shown. We note that the relative lens positions and caustics vary in time due to the orbital motion of the lens and thus we mark the positions at two different moments.

For both events, we find that the dominant second-order effect is the lens orbital motion. The dominance of the orbital effect is evidenced by the fact that the models considering only the orbital effect result in fits as good as those considering both the parallax and orbital effects. It is found that the consideration of orbital effects improves the fits by $\Delta \chi^{2}=1124.4$ and 158.4 compared to the standard models of OGLE-2006-BLG277 and OGLE-2012-BLG-0031, respectively. However, the improvements made by additionally considering the parallax effect are merely $\Delta \chi^{2}=16.3$ and 2.5 for the individual events.

To be noted is that parallax effects can mimic orbital effects to some extent for both events. We find that the improvements of the fits by the parallax effect are $\Delta \chi^{2}=841.6$ (cf. $\Delta \chi^{2}=1124.4$ improvement by the orbital effect) and 150.1 (cf. $\Delta \chi^{2}=158.4$ by the orbital effect) for OGLE-2006-BLG-277 and OGLE2012-BLG-0031, respectively. In addition, the values of the lens parallax determined without considering orbital effects substantially differ from those determined by considering orbital effects. This can be seen in Figure 6 where we present $\chi^{2}$ distributions in the space of the parallax parameters. For OGLE2006-BLG-277, the measured lens parallax is $\pi_{\mathrm{E}}=1.13 \pm$ 0.16 when both parallax and orbital effects are considered, 

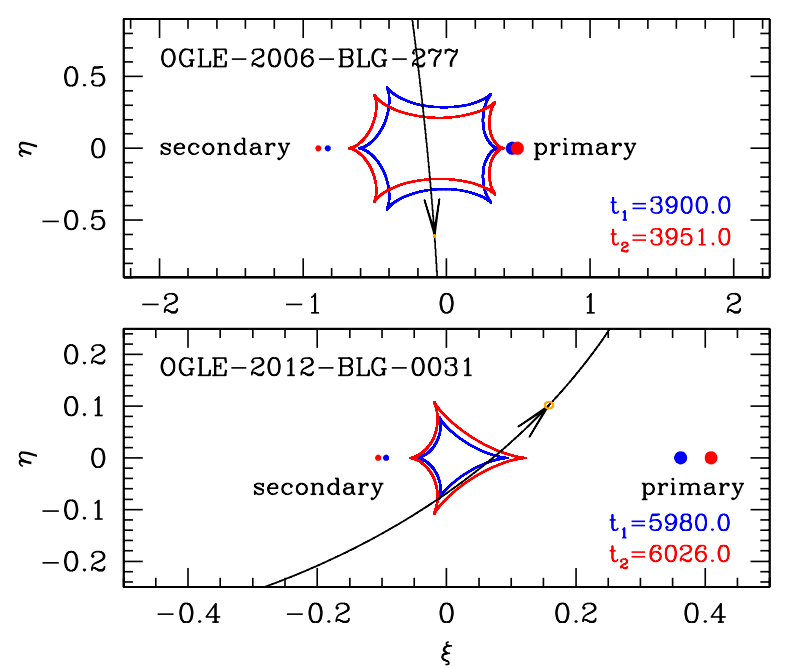

Figure 5. Geometry of the best-fit models for OGLE-2006-BLG-277 (upper panel) and OGLE-2012-BLG-0031 (lower panel). The small dots and closed solid curves represent the lens positions and caustics at two different times $t_{1}$ and $t_{2}$. The black solid curves with arrows represent source trajectories. The size of the small empty circle at the tip of the arrow of each source trajectory represents the source size. The abscissa and ordinate are parallel with and perpendicular to the binary axis, respectively. All lengths are normalized by the Einstein ring radius.

(A color version of this figure is available in the online journal.)

while $\pi_{\mathrm{E}}=0.70 \pm 0.05$ when only the parallax effect is considered. For OGLE-2012-BLG-0031, modeling considering only parallax effects results in a lens parallax $\pi_{\mathrm{E}}=0.31 \pm 0.08$ while the lens parallax is consistent with zero at the $3 \sigma$ level in the model considering additional orbital effects. These facts imply that orbital effects can masquerade as parallax effects and thus lens parallax values measured based on modeling that does not consider orbital effects can result in wrong values. This leads to wrong determinations of physical lens parameters because masses and distances to lenses are determined from measured values of the lens parallax.

It was pointed out by Batista et al. (2011) and Skowron et al. (2011) that the parallax component perpendicular to the relative lens-source motion, $\pi_{\mathrm{E}, \perp}$, is strongly correlated with the orbital parameter $d \alpha / d t$, causing a degeneracy between $\pi_{\mathrm{E}, \perp}$ and $d \alpha / d t$. They argued that this degeneracy occurs because the lens-source motion in the direction perpendicular to the Sun-Earth axis induces deviations in lensing light curves similar to those induced by the rotation of the binary-lens axis. For both events, OGLE-2006-BLG-277 and OGLE-2012-BLG-0031, the direction of the relative lens-source motion is similar to an east-west direction, and thus $\pi_{\mathrm{E}, \perp} \sim \pi_{\mathrm{E}, N}$. According to this degeneracy, the lens parallax vectors estimated by the "parallax only" and the "orbit + parallax" models should result in similar values of $\pi_{\mathrm{E}, E}$ while values of $\pi_{\mathrm{E}, N}$ can be widely different. However, both events analyzed in this work do not conform to the previous prediction. This implies that the parallax-orbit degeneracy is much more complex than previously thought, and thus it is essential to study the degeneracy in all cases where higher-order effects are detected.

We determine the physical lens parameters based on the bestfit solutions (orbit + parallax models). For this, we first determine the Einstein radius. The Einstein radius is determined by $\theta_{\mathrm{E}}=\theta_{*} / \rho_{*}$, where the normalized source radius $\rho_{*}$ is measured from the modeling and the angular stellar radius is determined based on the source type. The measured Einstein radius
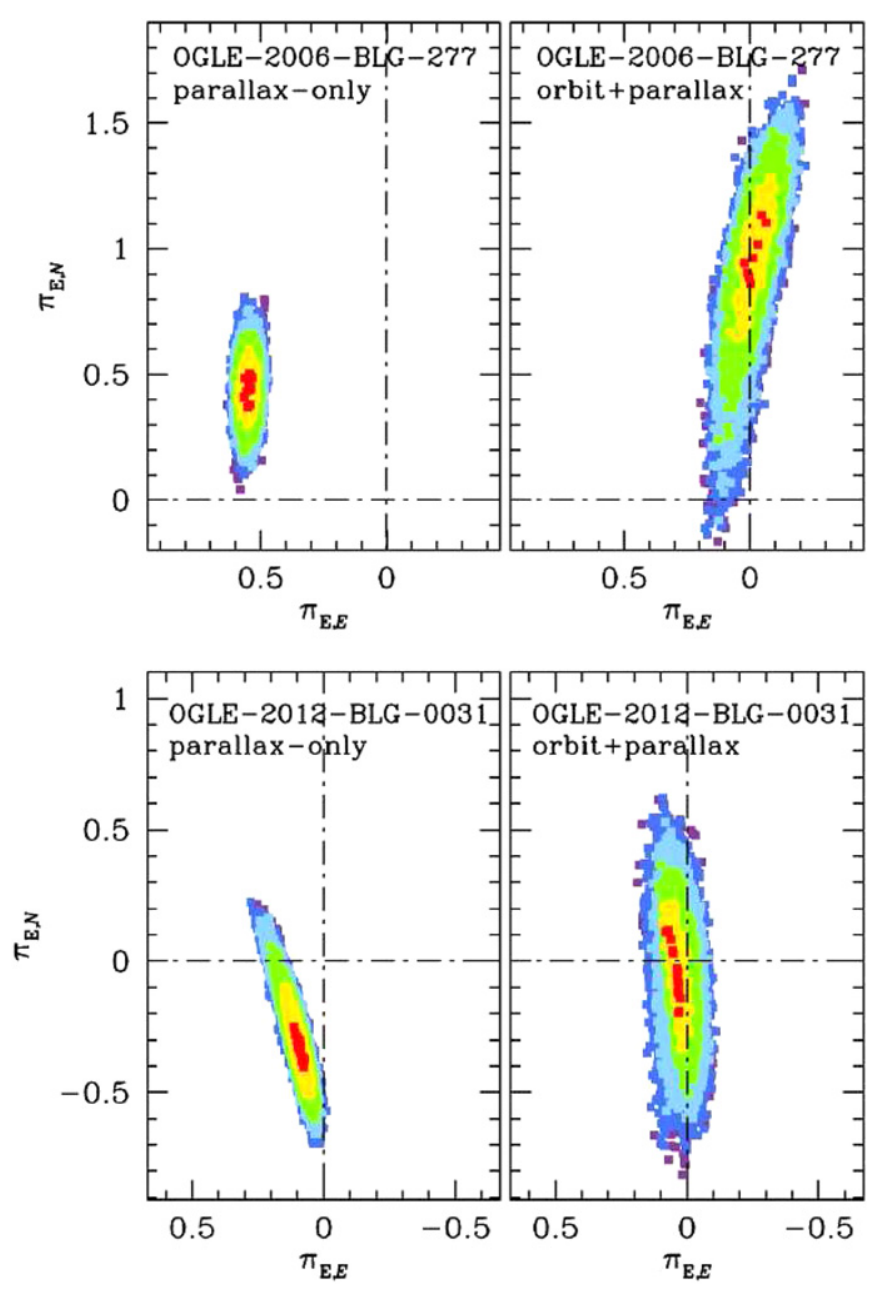

Figure 6. Distributions of $\chi^{2}$ in the space of the parallax parameters $\pi_{\mathrm{E}, E}$ and $\pi_{\mathrm{E}, N}$ for OGLE-2006-BLG-277 (upper panels) and OGLE-2012-BLG-0031 (lower panels). For each event, the distribution in the left panel is obtained from modeling considering only the parallax effect, while the distribution in the right panel is constructed by considering both the orbital and parallax effects. Different contours correspond to $\Delta \chi^{2}<1$ (red), 4 (yellow), 9 (green), 16 (sky blue), 25 (blue), and 36 (purple), respectively.

(A color version of this figure is available in the online journal.)

of the lens of OGLE-2006-BLG-277 is $\theta_{\mathrm{E}}=1.35 \pm 0.12$ mas. This corresponds to a relative lens-source proper motion of

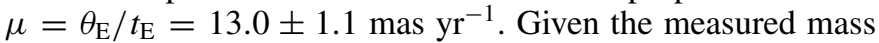
ratio between the lens components, the masses of the individual lens components are $M_{1}=M_{\text {tot }} /(1+q)=0.049 \pm 0.014 M_{\odot}$ and $M_{2}=q M_{\mathrm{tot}} /(1+q)=0.097 \pm 0.027 M_{\odot}$, respectively. Therefore, the lens is composed of a low-mass star and a brown dwarf. The distance to the lens is $D_{\mathrm{L}}=0.60 \pm 0.14 \mathrm{kpc}$. The close distance explains the relatively high proper motion

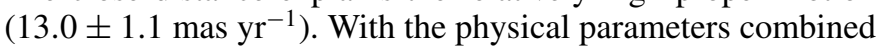
with orbital parameters, we evaluate the ratio of transverse kinetic to potential energy

$$
\left(\frac{\mathrm{KE}}{\mathrm{PE}}\right)_{\perp}=\frac{\left(r_{\perp} / \mathrm{AU}\right)^{2}}{8 \pi^{2}\left(M_{\mathrm{tot}} / M_{\odot}\right)}\left[\left(\frac{1}{s} \frac{d s}{d t}\right)^{2}+\left(\frac{d \alpha}{d t}\right)^{2}\right],
$$

where $r_{\perp}$ denotes the projected binary separation (Dong et al. 2009). The ratio should obey $(\mathrm{KE} / \mathrm{PE})_{\perp} \leqslant \mathrm{KE} / \mathrm{PE}<1$ for kinetically stable binary orbit. We find $(\mathrm{KE} / \mathrm{PE})_{\perp}=0.20 \pm 0.04$. For OGLE-2012-BLG-0031, it is difficult to determine the physical lens parameters not only because the source type is 

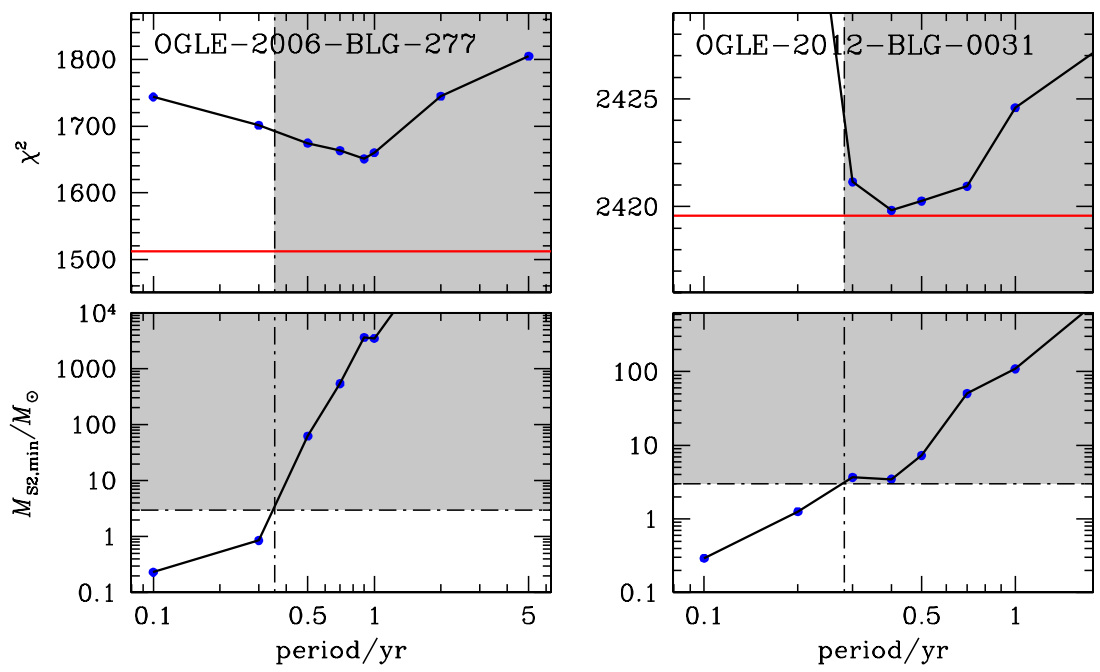

Figure 7. Results of the xallarap modeling for OGLE-2006-BLG-277 (left panels) and OGLE-2012-BLG-0031 (right panels). The upper panels show $\chi^{2}$ distributions as a function of the orbital period of the source star, $P$. The lower panels show the lower limit of the source companion's mass as a function of $P$. The straight red line in the upper panels represents $\chi^{2}$ of the best-fit orbital + parallax model. The shaded area indicates that the models are ruled out by the companion's mass constraint. (A color version of this figure is available in the online journal.)

uncertain but also because the lens parallax is consistent with zero.

\section{SUMMARY AND CONCLUSION}

We analyzed two binary-lens events, OGLE-2006-BLG-277 and OGLE-2012-BLG-0031, for which the light curves exhibit significant residuals from standard binary-lens models. From modeling considering higher-order effects, we found that the residuals were greatly removed by considering the effect of the lens orbital motion. We also found that parallax effects could mimic orbital effects to some extent and parallax values measured without considering the orbital effect could result in dramatically different values from true ones, and thus wrong determinations of the physical lens parameters. We also found that the lens of OGLE-2006-BLG-277 was a binary composed of a low-mass star and a brown dwarf companion.

Work by C.H. was supported by the Creative Research Initiative Program (2009-0081561) of the National Research Foundation of Korea. A.G. was supported by NSF grant AST 1103471. The OGLE project has received funding from the European Research Council under the European Community's Seventh Framework Programme (FP7/2007-2013)/ERC grant agreement No. 246678 to A.U. S.D. was supported through a Ralph E. and Doris M. Hansmann Membership at the IAS and NSF grant AST-0807444. D.M.B., M.D., M.H., R.A.S., and Y.T. thank the Qatar Foundation for support from QNRF grant NPRP-09-476-1-078. Dr. David Warren gave financial support to Mt. Canopus Observatory.

\section{APPENDIX}

\section{XALLARAP EFFECTS}

Besides the orbital motion of the observer and lens, the orbital motion of the source star can also affect the relative lens-source trajectory if the source is a binary (Griest \& Hu 1992; Han \& Gould 1997; Dominik 1998). The latter effect is often referred to as the "xallarap effect" (parallax spelled backwards).

To double check our analysis, we conduct additional modeling considering the xallarap effect. To describe the effect, one must include five parameters: the orbital period $P$, the phase angle $\varphi$, and inclination $i$ of the orbit, and the north and east components of the xallarap vector, $\xi_{\mathrm{E}, N}$ and $\xi_{\mathrm{E}, E}$ (Dong et al. 2009).

In Figure 7, we present the result of xallarap analysis. For each event, the upper panel shows $\chi^{2}$ as a function of source orbital period. The lower panel shows the minimum mass of the unseen companion to the source, $M_{\mathrm{S} 2 \text {, min }}$, with respect to the orbital period. The value $M_{\mathrm{S} 2 \text {, min }}$ is set by

$$
M_{\mathrm{S} 2, \min }=\frac{\left(\xi_{\mathrm{E}} \hat{r}_{\mathrm{E}} / \mathrm{AU}\right)^{3}}{(P / \mathrm{yr})^{2}} M_{\odot},
$$

where $\xi_{\mathrm{E}}=\left(\xi_{\mathrm{E}, N}^{2}+\xi_{\mathrm{E}, E}^{2}\right)^{1 / 2}$, and $\hat{r}_{\mathrm{E}}$ is the physical Einstein radius projected onto the source plane (Dong et al. 2009; Kains et al. 2013).

For OGLE-2006-BLG-277, it is found that the best-fit xallarap solution yields a model that is worse than the best-fit orbital + parallax model with $\Delta \chi^{2} \sim 139$, and thus the xallarap interpretation is excluded with a significant confidence level. For OGLE-2012-BLG-0031, on the other hand, the xallarap model yields a solution with $\chi^{2}$ equivalent to that of the orbital + parallax model $\left(\Delta \chi^{2} \sim 0.2\right)$. However, the estimated lower-mass limit of the companion is $\geqslant 3 M_{\odot}$. If the companion is a regular star, it corresponds to an A type and earlier, but such an early type star is extremely rare in the Galactic bulge. Thus, the only explanation is that the companion is a dark object such as a black hole, but this is unlikely. Therefore, we also rule out a xallarap interpretation of the event.

\section{REFERENCES}

Alard, C., \& Lupton, R. H. 1998, ApJ, 503, 325

Albrow, M. D., Beaulieu, J.-P., Birch, P., et al. 1998, ApJ, 509, 687

Albrow, M. D., Beaulieu, J.-P., Caldwell, J. A. R., et al. 2000, ApJ, 534,894

Albrow, M. D., Horne, K., Bramich, D. M., et al. 2009, MNRAS, 397, 2099

An, J. H. 2005, MNRAS, 356, 1409

An, J. H., Albrow, M. D., Beaulieu, J.-P., et al. 2002, ApJ, 572, 521

Batista, V., Gould, A., Diester, S., et al. 2011, A\&A, 529, 102

Bennett, D. P., Rhie, S. H., Becker, A. C., et al. 1999, Natur, 402, 57

Bramich, D. M. 2008, MNRAS, 386, 77 
Choi, J.-Y., Shin, I.-G., Park, S.-Y., et al. 2012, ApJ, 751, 41

Claret, A. 2000, A\&A, 363, 1081

Dominik, M. 1998, A\&A, 329, 361

Dominik, M. 1999, A\&A, 349, 108

Dong, S., Depoy, D. L., Gaudi, B. S., et al. 2006, ApJ, 642, 842

Dong, S., Gould, A., Udalski, A., et al. 2009, ApJ, 695, 970

Gould, A. 1992, ApJ, 392, 442

Gould, A. 1994, ApJ, 421, 71

Gould, A. 2004, ApJ, 606, 319

Gould, A. 2008, ApJ, 681, 1593

Gould, A., Udalski, A., An, D., et al. 2006, ApJ, 644, 37

Griest, K., \& Hu, W. 1992, ApJ, 397, 362

Griest, K., \& Safizadeh, N. 1998, ApJ, 500, 37

Han, C., \& Gould, A. 1997, ApJ, 480, 196

Jaroszyński, M., Skowron, J., Udalski, A., et al. 2010, AcA, 60, 197
Jaroszyński, M., Udalski, A., Kubiak, M., et al. 2005, AcA, 55, 159

Jung, Y. K., Han, C., Gould, A., \& Maoz, D. 2013, ApJ, 768, 7

Kains, N., Street, R. A., Choi, J.-Y., et al. 2013, A\&A, 552, 70

Kayser, R., Refsdal, S., \& Stabell, R. 1986, A\&A, 166, 36

Mao, S., \& Paczyński, B. 1991, ApJ, 374, 37

Pejcha, O., \& Heyrovský, D. 2009, ApJ, 690, 1772

Schneider, P., \& Weiss, A. 1986, A\&A, 164, 237

Skowron, J., Udalski, A., Gould, A., et al. 2011, ApJ, 738, 87

Shin, I.-G., Sumi, T., Udalski, A., et al. 2013, ApJ, 764, 64

Shin, I.-G., Udalski, A., Han, C., et al. 2011, ApJ, 735, 85

Tsapras, Y., Street, R., Horne, K., et al. 2009, AN, 330, 4

Udalski, A. 2003, AcA, 53, 291

Wambsganss, J. 1997, MNRAS, 284, 172

Witt, H. J., \& Mao, S. 1994, ApJ, 430, 505

Woźniak, P. R., Udalski, A., Szymański, M., et al. 2001, AcA, 51, 175 